\title{
Toward Semantic Web Services for Multimedia Adaptation
}

\author{
Dietmar Jannach, Klaus Leopold, Christian Timmerer, and Hermann Hellwagner
}

\author{
Department of Business Informatics \& Application Systems, \\ Department of Information Technology \\ University Klagenfurt, 9020 Austria \\ \{firstname. lastname\}@uni-klu.ac.at
}

\begin{abstract}
Universal Multimedia Access (UMA), where users can consume any multimedia resource anywhere at any time, is the driving vision of ongoing ISO/IEC Moving Picture Experts Group (MPEG) standardization efforts. In that context, intelligent adaptation means that before resources are sent over the network, they are prepared according to the client's device capabilities, the network conditions, or even the user's content preferences. In this paper, we argue that Semantic Web Services can serve as a key enabling technology to achieve the goals of UMA. As the standards evolve, more and more specialized software tools will be available that provide specific functionalities for adapting the media in different dimensions. When the functionality of such tools is described declaratively with the means of Semantic Web Services technology, intelligent adaptation network nodes can be developed, capable of automatically composing multi-step adaptation sequences and dynamically integrating such services available on the Web. This paper describes the architecture and a prototypical implementation of an intelligent adaptation node that supports automatic, knowledge-based service composition which is made possible by the shared domain ontology defined in MPEG metadata standards.
\end{abstract}

\section{Introduction}

The increasing availability of high-speed wired and wireless networks as well as the development of a new generation of powerful (mobile) end-user devices like Personal Digital Assistants or cell phones leads to new ways in which multimedia resources can be consumed over the Web. At the same time, new standards like MPEG-7 [1] have become available, allowing us to enrich media content with semantic content annotations, which in turn facilitates new forms of multimedia experience, like search on specific topics or semantics-based content selection and filtering.

MPEG-21 is an emerging ISO/IEC standard that aims at addressing these new challenges by defining a normative open framework for multimedia delivery and consumption involving all parties in the delivery and consumption chain ${ }^{1}$. One of the driving visions of these efforts is Universal Multimedia Access (UMA), where users can consume any multimedia resource anywhere at any time. A major part of these

\footnotetext{
${ }^{1}$ http://www.chiariglione.org/mpeg
} 
standardization efforts deals with the definition of an interoperable framework for Digital Item Adaptation (DIA) [2]. Adaptation in that context means that a given resource is adapted according to the user's specific requirements as well as to given device and network capabilities before it is sent over the network. Figure 1 shows a typical distributed environment where different types of end-user devices are used to consume multimedia content over heterogeneous networks.

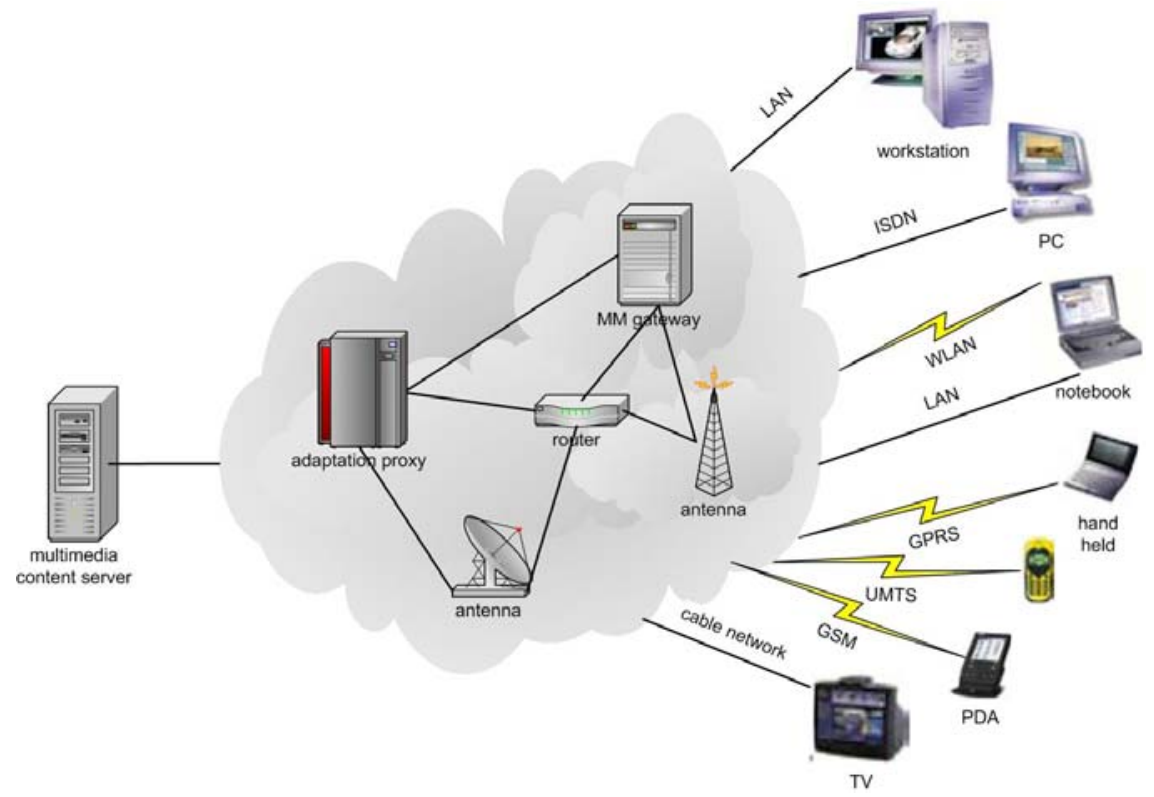

Fig. 1. Multimedia provision over the Internet

Currently, the scope of MPEG-21 standards with respect to adaptation comprises mechanisms (normative description schemes) enabling interoperability by describing the adaptation problem, like the terminal capabilities, the user preferences, or the format of the resource itself. The implementation of an engine that actually performs the required adaptation steps is left to tool vendors and is currently beyond the scope of standardization.

Nonetheless, there is a specific requirement in that context that is only marginally addressed in the current standardization efforts, i.e., the multimedia framework "... shall support a convenient and efficient way to concatenate multiple adaptation steps" [6]. Therefore, in order to enable efficient treatment and automatic construction of such transformation sequences by an adaptation engine, we argue that mechanisms are needed that allow us to capture the semantics of adaptation steps. Note that it does not seem realistic that one single software tool will be able to perform all required adaptation steps for the various user preferences, terminal capabilities, network characteristics, or even for the diverse set of coding formats. Consequently, such a mechanism has to be expressive enough and independent from the actual 
implementation such that interoperability among tools of different vendors is guaranteed. Finally, an open and extensible approach has to be chosen, such that no changes in the general mechanism are required when new forms of adaptation are possible as the standards evolve or new tools become available.

In this paper, we demonstrate how Semantic Web Services [5],[7] can serve as a key enabling technology for fulfilling these currently open requirements. The contribution of this work is twofold. First, semantic descriptions of adaptation steps allow us to build new forms of adaptation servers capable of intelligently transforming multimedia resources according to the individual user's demands and thus providing enhanced multimedia experience over the Internet.

Second, the application domain is also an ideal test bed for current Semantic Web Services technology in a realistic, real-world problem setting. It also shows that the availability of a shared domain ontology is the key factor for its practical applicability, which in our case is given indirectly by the terms, the vocabulary, and the semantics defined in the existing MPEG standards.

\section{Semantic Web Services}

The idea of the Semantic Web [5] is to have resources available on the Web that can be retrieved by content and not by keyword [9] and can also be automatically accessed by software applications or agents. Services in that context are specific "resources" where the invocation of such a service typically results in a world-altering action, like the sale of a book. In order to facilitate these new forms of using the Web, the available resources have to be semantically annotated, which leads to the development of new markup languages. OWL [8], [9] is the most prominent approach having the status of a W3C recommendation. Languages like OWL can be both used for creating ontologies for a domain as well as in the descriptions of particular Web sites.

If we look at service-type resources, the envisioned automation in the Semantic Web means that we need annotation mechanisms such that software agents can, e.g., search for appropriate services, invoke them with the required parameters, or even compose a complex transaction from several distributed services. A typical example from the domain of travel planning is described in [7], where a complete travel arrangement requires the invocation of multiple services, like making a hotel reservation or booking a flight. These requirements are currently addressed in OWL-S [9], which constitutes a general ontology for the domain of services.

Automatic service composition and interoperation are the most important aspects with respect to our problem domain of intelligent multimedia adaptation. (Other OWL features related to organizational aspects, e.g., service registration and publication, are not discussed in this paper.) In order to support these tasks, OWL-S comprises mechanisms to describe the functionality of a service in terms of a description of the transformation that is caused when a service is invoked. In particular, with OWL-S one can declaratively describe the inputs, outputs, preconditions, and effects (IOPE) of a service. This particular IOPE approach is also widely used for planning tasks in Artificial Intelligence. 
The Semantic Web and its underlying technologies can therefore also change the way software is developed in a more general way, i.e., as Semantic Web proponents put it, "creating software will be just a matter of finding the right components on the Web and a specification document linking to these components. The new software is again just a resource available on the Web"2.

\section{An Adaptation Framework Based on Semantic Web Services}

The new possibilities of Digital Item Adaptation in the context of the MPEG-7 and MPEG-21 standards induce new requirements for multimedia servers which should be able to intelligently adapt the media resources according to their clients' needs:

- The system should be able to perform adaptation on various resource types (videos, images, etc.) and with respect to different dimensions, e.g., media transcoding or content selection. At the moment no tool exists that can handle all these adaptations in a single step. Therefore, multi-step adaptation chains have to be supported.

- A lot of adaptation utilities with specific functionality are already (freely) available and should be re-used by the adaptation engine. As such, the adaptation engine must provide open interfaces such that these tools can be easily incorporated.

- As the standards emerge and the corresponding tools are available, additional coding formats and forms of adaptation will become possible. Consequently, the mechanism for computing the required adaptation operations should be based on declarative specifications in order to be robust and extensible with respect to such additions.

In order to address these new requirements, we propose a knowledge-based approach [3], which is sketched in Figure 2. Here, a client requests a certain resource from a multimedia server and also provides a declarative description of its usage environment (i.e., device capabilities, network conditions, and user preferences). Together with the multimedia resource, the media server stores descriptions of the content and the available adaptation tools. These metadata are the input for the adaptation decision taking engine which produces a sequence of appropriate transformation steps (an adaptation plan). The adaptation plan is then forwarded to the adaptation engine which executes the adaptation tools on the original resource according to the adaptation plan and, thus, produces the adapted multimedia resource.

In the framework presented in this paper, we propose to model this process of constructing the adaptation plan as a classical state-space planning problem (see, e.g., [10]), where the description of the existing resource is the start state, the functionality provided by existing tools corresponds to world-altering actions, and the user requirements can be mapped to the goal state.

\footnotetext{
$2 \mathrm{http}: / / \mathrm{www} \cdot$ semanticweb.org
} 


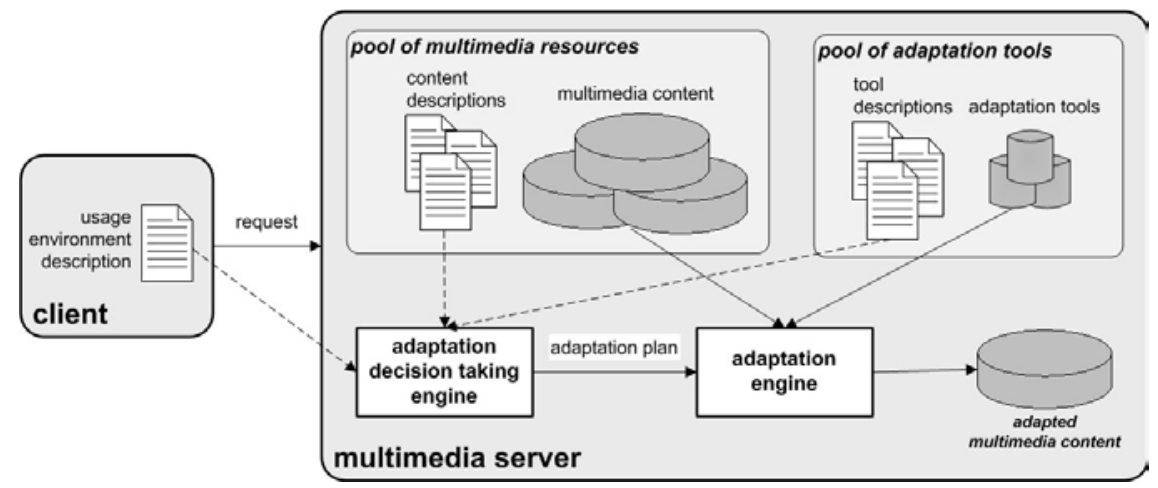

Fig. 2. Overview of knowledge-based adaptation

The following simple example sketches how transformation steps can be described in terms of inputs, outputs, preconditions, and effects (IOPE) and how a suitable adaptation plan for a given problem is constructed. We use a simplified logic notation instead of the XML notation for better readability [4].

The existing resource is described by the following Prolog like facts:

coding_format(mpeg_4_Visual_ES). color_domain(true).frame_size $(640,480)$.

The terminal's capabilities are as follows:

decoding_capabilities(mpeg_1).color_capabilities(false).display_size(320, 240).

Let us assume that the adaptation steps grey scaling and spatial scaling are available in an existing image transformation API, among others, such as encoding and decoding. Note that the adaptation operations as described in the following, are performed on a single image of a video. The decomposition of a video into frames is done by another tool executed by the adaptation engine.

Grey scaling (greyscale):

Input: image

Output: $\quad$ greyimage

Preconditions: yuvImage(image), color(true).

Effects: $\quad$ yuvImage(greyimage), color(false).

Spatial scaling (spatialscale):

Input: $\quad$ image, $x, y$, newx, newy

Output: $\quad$ scaledimage

Preconditions: yuvImage(image), width(x), height(y)

Effects: $\quad y u v I m a g e($ image), width(x), height(y), horizontal(newx), vertical(newy).

A corresponding plan can be computed by a standard state-space planner as follows, where "fb1" to "fb5" ("frame buffer") are the unified variables:

1: decode(fb1,mpeg_4_visual_es,fb2)

2: spatialscale (fb2, $6 \overline{4} 0,480,320,240, \mathrm{fb} 3)$

3: greyscale $(\mathrm{fb} 3, \mathrm{fb} 4)$

4: encode $(\mathrm{fb} 4$, mpeg_1,fb5) 
Using the IOPE approach and a logic language for modeling the functionality of adaptation services has two main advantages. First, the general approach is simple to comprehend and has a defined, commonly agreed semantic such that the automated composition of action sequences is possible. In addition, the approach is flexible, since the core planner operates on arbitrary symbols, such that new types of predicates or new actions can be easily added when they are available, without changing the implementation. Finally, there is a long history in state-space planning that showed that IOPE-style descriptions are expressive enough for a wide range of problem domains.

Note that in our specific problem setting, the IOPE modeling approach can also serve as a means for addressing further domain-specific challenges. First, as sketched in the example, frame width and height are used for describing the size of the existing resource; the client's terminal capabilities, however, are described in terms of the display size, i.e., vertical and horizontal resolution as shown in Document 1 and Document 2. Such a situation is quite common in the problem domain, as for instance different multimedia standards are involved. Such a situation can be coped with as described in the example, i.e., the ontological mapping between those terms or other (mathematical) relations can be explicitly modeled using preconditions and effects of the actions. Second, adaptation is not necessarily limited to the transformation of the media resource itself, but the same mechanism can be utilized to transform the accompanying (semantic) content descriptions, if this is needed by the client. In other words, in the adaptation process also the metadata may be updated correctly.

OWL-S 1.0 does not yet specify the rule language to be used for describing conditions or outputs of actions. Different candidates like Semantic Web Rule Language $^{3}$ are currently evaluated and will soon be part of OWL-S, as the common language and defined semantics are crucial for interoperability. However, for our problem domain we feel that in typical cases no unusual requirements on the expressiveness of the language should arise that are not within the scope of the current proposals. Another interesting aspect in our problem domain is that the level of detail of the functional descriptions of available adaptation services can vary. In the example given, each action is an atomic, single-step picture transformation. This finegranular specification is reasonable in cases when, e.g., open-source transcoding software like FFMPEG or ImageMagick ${ }^{4}$ should be re-used in the adaptation engine. In this scenario, the adaptation chain and the execution plan is composed of API calls to a local media processing library, showing that existing libraries can easily be integrated. On the other hand, as newer standards for semantic content annotation like MPEG-7 are increasingly established in commercial environments, specialized software companies will be able to provide such advanced adaptation functionality as (chargeable) services for their clients. With the approach described in this paper, however, the potential distributed nature of the individual services is transparent for the engine.

In our opinion, one of the most important aspects that can hamper the usage of Semantic Web Services is the problem of having a shared domain ontology. If we think again of the well-known travel planning problem, automatic service

\footnotetext{
${ }^{3}$ http://www.w3.org/Submission/2004/SUBM-SWRL-20040521/

${ }^{4} \mathrm{http}: / /$ ffmpeg.sourceforge.net, http://www.imagemagick.org
} 
composition can only be done if all participants not only use the same terms like "hotel reservation", but also associate the same meaning with that term. Semantic markup languages like OWL (or its predecessor DAML+OIL) only provide a common infrastructure for defining, integrating, and reasoning about such ontologies, but cannot solve the problem of agreeing on a domain-specific ontology in a community. In fact, even for well-understood domains like business-to-business electronic commerce or electronic supply-chain management, several competing pseudo-standards for XML-based data exchange emerged ${ }^{5}$. In our domain, however, such a common understanding can be reached by interpreting the existing MPEG standards as the basis for describing the functionality and semantics of the adaptation services.

\section{Multimedia Standards as Domain Ontology}

Multimedia standards like MPEG-7 or MPEG-21 precisely specify the way how multimedia resources and usage environments (e.g., terminal and network capabilities) can be annotated with additional information. XML Schema ${ }^{6}$ technology is extensively used in the standards as the specification language for the shared vocabulary. Specific media resources are annotated with document instances that correspond to that standardized schema. Document 1 shows an example of how a video resource is annotated in MPEG-7; in particular it specifies the parameters color or the frame size, which we used in the planning example.

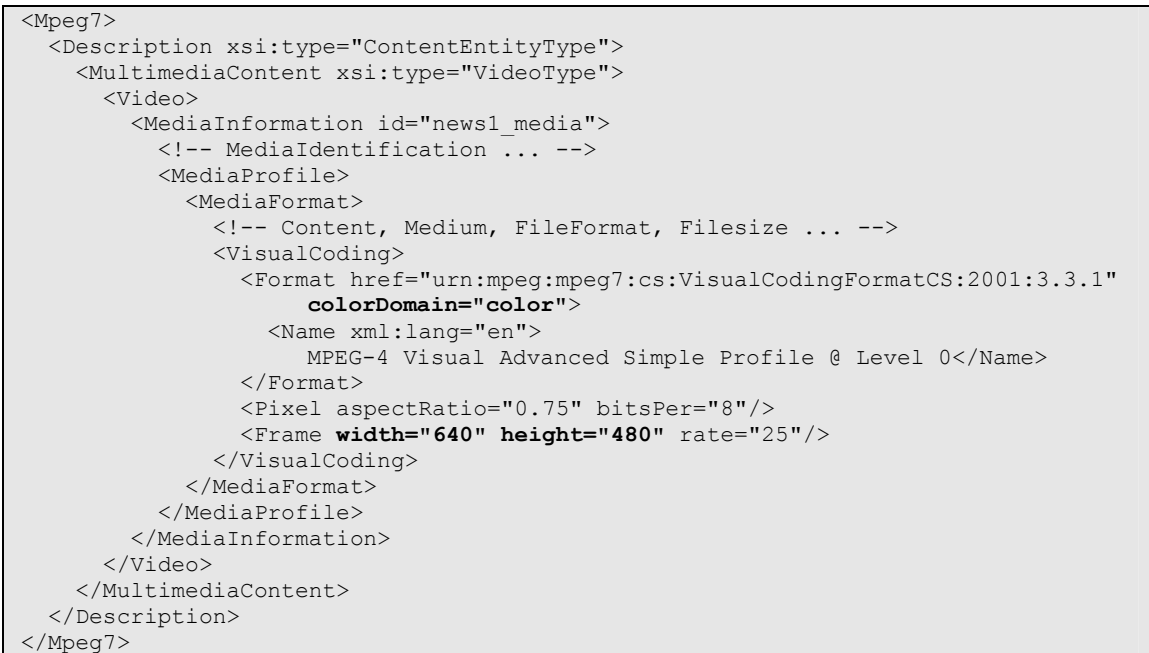

Document 1. Fragment of an MPEG-7 video description

\footnotetext{
${ }^{5} \mathrm{http} / / / \mathrm{www} . \mathrm{ebxml.org} /$

${ }^{6} \mathrm{http}: / /$ www.w3.org/XML/Schema
} 
The MPEG-21 DIA description of the end device used in the planning example is shown in Document 2.

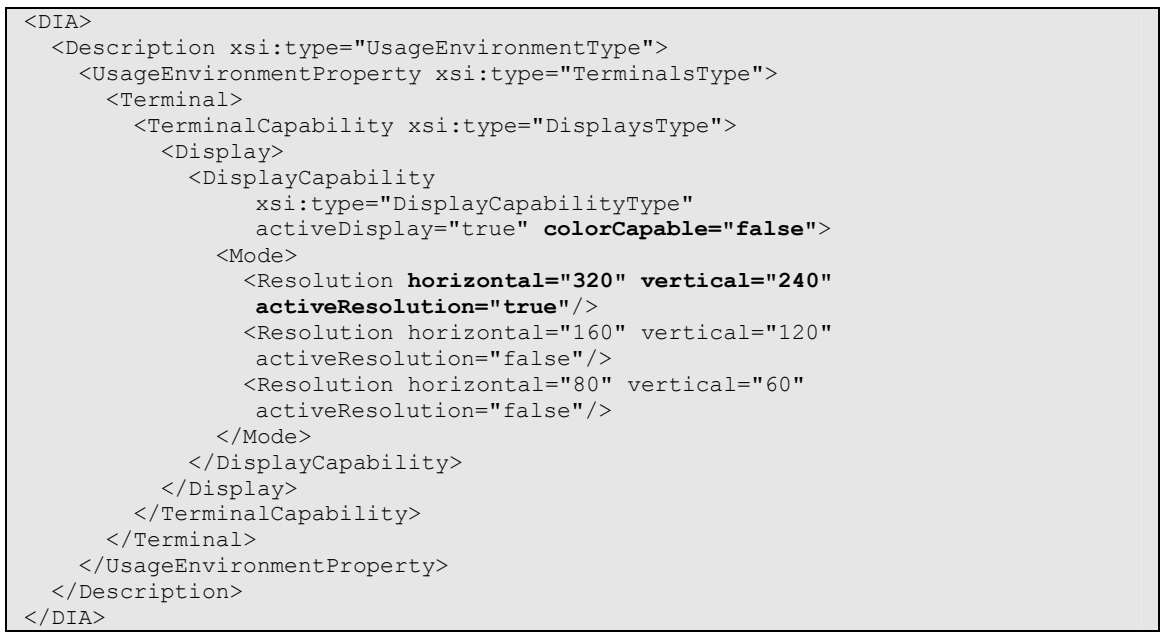

Document 2. MPEG-21 DIA description of a terminal with certain display capabilities

Although not explicitly mentioned as such, the definitions in the standards implicitly form a precise ontology for the multimedia domain. The dimensions and corresponding syntactical structures in which a resource can be annotated are strictly defined. The intended semantics of the terms is specified in natural language. For the examples above, the colorDomain attribute describes the color domain of the video with a frame size of $640 \times 480$ pixels indicated by the width and height attributes. The resolution and color capabilities of the rendering device are described by the horizontal and vertical attributes and the colorCapable attribute respectively. Please note that such a rendering device could support multiple resolutions and the resolution mode currently used is indicated by the Boolean activeResolution attribute.

For our multi-step adaptation problem, the representation mechanism for the resource descriptions (MPEG-7) and the client preferences (MPEG-21) is therefore already given. At the moment, the language to be used for describing the semantics of the transformation services is not within the scope of OWL-S. Quite obviously, the concepts that are used in pre-conditions and effects of the services have to correspond to those that are used in the standards.

In our current prototypical implementation, we have chosen a proprietary notation that is automatically transformed to an internal, logic-based format which can be exploited by a Prolog-based planning engine. Our experiments show that the complexity of the conditions and expressions that we need for describing a transformation service is rather low and the description can be expressed by a set of facts. This also holds for a potential extension of the approach for the problem of 
Digital Rights Management $(D R M)^{7}$. If some transformations, like the extraction of the audio stream from a movie, require particular rights, such information can be easily incorporated in our extensible approach and encoded in the pre-conditions of the transformation functions.

Overall, we are optimistic that the rule language that is developed for OWL-S will be expressive enough for our problem domain and that compatibility of our prototype can be reached by transforming the descriptions on the syntactical level.

Note that a full re-implementation of the MPEG standards with the representation means of OWL-S is in principle possible and would allow for a consistent usage of OWL technology throughout. As a side effect, such an approach would in our opinion also improve the comprehensibility and clarity of the standards. A first step in that direction can be the construction of an OWL-based ontology for the domain, which can serve as accompanying material that can be used for constructing the semantics descriptions for adaptation services. Figure 3 illustrates a small fraction of the object relations within an MPEG-21 document which could be translated to OWL in a straightforward manner.

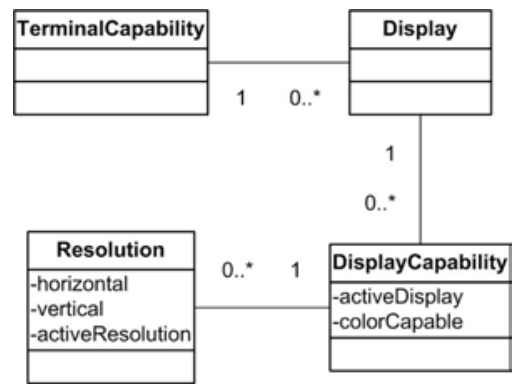

Fig. 3. Fraction of the object relations of an MPEG-21 document

\section{Experimental Results}

In order to evaluate the general feasibility of the described approach, we implemented a first proof-of-concept prototype. The architecture of our system corresponds to the system overview depicted in Figure 2. The intelligence required for computing the adaptation chains is implemented in a light-weight state-space planner written in SWI-Prolog ${ }^{8}$. Beside a small module responsible for actually executing the needed transformations on the original resource, the system consists of parsers and Javabased transformation tools for processing the XML documents and transforming them to the internal representation. With regard to computational complexity, we found that the actual planning problems were rather simple and did not cause performance problems. In particular the length of the needed plans, which is an important factor

7 Open Mobile Alliance (http://www.openmobilealliance.org) and Digital Media Project (http://www.chiariglione.org/project)

${ }^{8} \mathrm{http}: / /$ www.swi-prolog.org 
influencing the complexity of the problem space, was very small, typically no more than five to seven steps. In all of our experiments, the computation of the plan could be done in less than one second on a standard PC or the non-existence of such a plan could be proven. In addition, the planning process only takes a marginal part of the overall process of providing an adapted resource over the Web. The costly operations are typically the transformation itself or the establishment of streaming sessions, in particular when network conditions change or session mobility is involved, which means that during the resource consumption the session is migrated to another enduser device.

At the moment, we have not yet performed experiments in a real distributed environment, but rather integrated locally available transformation tools like FFMPEG or ImageMagick. Nonetheless, the usage of declarative functionality descriptions and a general function invocation mechanism already provided significant benefits. For instance, the system is extensible as the incorporation of new transformation tools does not cause any implementation efforts; in addition, interoperability across platforms and programming languages is guaranteed as the actual tools provide an independent, XML-based invocation interface based on Web Service technology. Finally, for the implementation of a scenario where multiple adaptation servers are used that only conjointly can perform all required adaptation steps, we can then rely on existing and well-established technologies like WSDL or $\mathrm{UDDI}^{9}$ for administrative tasks like service registration etc.

\section{Related Work}

Up to now, Semantic Web technology was not broadly used in the field of current multimedia-related research and only limited efforts like in [11] where spent in the direction of bringing together tools and technologies for describing multimedia content and the shared knowledge representation provided by the Semantic Web.

In [12] for instance, an approach towards automated, knowledge-assisted content analysis is described. In this work, the authors show how domain specific ontologies can be used for narrowing the semantic gap that arises when concepts and objects should be automatically extracted without human intervention from image or spatiotemporal features. They use Semantic Web technologies as a representation mechanism for modeling additional domain-specific knowledge because of the intention that the general mechanisms of knowledge representation and reasoning should be applicable for arbitrary other domains.

In [13] an approach is presented, where ontologies are serving as integrating technology for advanced concept, metadata, and content browsing and retrieval in the domain of digital museum information. In this work, a domain ontology and a semantic layer with references to the actual objects of the digital collection like 2-D or 3-D images and models was developed. Semantic Web technology was mainly used for the purpose of system integration, as the existing information like textual metadata on items is often stored in separate collections and legacy systems.

\footnotetext{
${ }^{9}$ http://www.w3.org/TR/wsdl, http://www.uddi.org
} 
The described approaches use Semantic Web technology basically as a tool for further semantic annotations and domain-specific ontologies with the goals of better retrieval methods or content analysis. In our work, we use Semantic Web technologies at a different level as we aim at transforming the contents themselves. The shared ontology required for the integration of such adaptation services is in our case domain-independent and defined in the existing multimedia standards. As such, we also view the problem domain as a promising area for Semantic Web Services in general, as the number of reported real-world application fields is still limited.

In [14] the architecture of an adaptive proxy for MPEG-4 visual streams is described which adapts MPEG-4 resources according to terminal capabilities (display size and color capabilities) and network characteristics (available bandwidth). Therefore, an adaptor chain concept has been introduced enabling the concatenation of several adaptation steps. However, this approach is currently restricted to the MPEG-4 visual domain. While an adaptor chain is dynamically instantiated according to the usage environment, the approach lacks extensibility in the sense that new adaptors must implement a predefined interface and need to be integrated into the existing system by re-compilation of the whole adaptation engine. The concept of adaptor chains follows an imperative (procedural) approach whereas our approach is declarative and therefore not restricted to a certain programming language, enabling the integration of new adaptation algorithms at run-time.

Within MPEG-21, only one tool (AdaptationQoS) which is part of the DIA specification deals somehow with the issue of adaptation decision taking. AdaptationQoS supports the selection of optimal parameter settings for media resource adaptation engines that satisfy constraints imposed by terminals and/or networks while maximizing the Quality of Service [15]. Therefore, the relationship between constraints, feasible adaptation operations satisfying these constraints, and associated utilities (qualities) is described for a certain resource. However, these relationships do not cope with the semantics of the actual adaptation process as described within this paper. Furthermore, AdaptationQoS provides only relationships for a subset of the possible adaptation operations and is always related to one particular resource. Thus, it reflects only the adaptation possibilities taken into account by the content creator or provider. On the other hand, this information can be used as a decision support mechanism by providing optimal parameter settings within one adaptation step. Additionally, it provides means for describing optimization problems allowing the adaptation engine to achieve suboptimal solutions which is currently not supported by our approach, but will become part of our future work.

\section{Conclusions}

In this work, we argued that Semantic Web Services technologies can serve as the technical infrastructure for future multimedia adaptation services. These technologies allow us to build a new generation of intelligent multimedia adaptation engines that fully exploit the new possibilities that arise with existing and upcoming multimedia annotation standards. With the help of semantic descriptions of the functionality of 
existing transformation tools and services, complex multi-step adaptation sequences can be automatically computed and executed on arbitrary media resources.

The employed mechanisms inherently support interoperability, openness, and extensibility such that independence from platforms, programming languages, or the tool implementation is guaranteed.

\section{References}

1. J. M. Martinez, R. Koenen, and F. Pereira, "MPEG-7 - The Generic Multimedia Content Description Standard, Part 1," IEEE MultiMedia 9(2):78-87, April-June 2002.

2. A. Vetro and C. Timmerer, "Overview of the Digital Item Adaptation Standard", IEEE Transactions on Multimedia, Special Issue on MPEG-21, 2004 (to appear).

3. D. Jannach, K. Leopold, and H. Hellwagner, "An extensible framework for knowledgebased multimedia adaptation”, in: B. Orchard, C. Yang, M. Ali (eds.): Innovations in Applied Artificial Intelligence, LNAI 3029, Springer Verlag 2004.

4. K. Leopold, D. Jannach, and H. Hellwagner, "Knowledge-based Media Adaptation", Proceedings of the SPIE ITCom 2004, Internet Multimedia Management Systems IV, vol. 5601, 25-28 October 2004 (to appear).

5. T. Berners-Lee, J. Hendler, and O. Lassila, "The Semantic Web", Scientific American 284(5):34-43, 2001.

6. J. Bormans (ed.), "MPEG-21 Requirements v 1.5", ISO/IEC JTC 1/SC 29/WG 11 N5873, Trondheim, July 2003.

7. S. McIlraith, T.C. Son, and H. Zeng, "Semantic Web Services", IEEE Intelligent Systems, Special Issue on the Semantic Web, 16(2):46-53, 2001.

8. D. L. McGuiness and F. van Harmelen (eds.), "OWL Web Ontology Language Overview", February 2004, http://www.w3.org/TR/owl-features/.

9. The OWL Services Coalition, "OWL-S: Semantic Markup for Web Services", Technical Report, 2004, http://www.daml.org/services/owl-s/1.0/.

10. I. Bratko, Prolog Programming for Artificial Intelligence, 3rd Edition, Addison-Wesley 2000.

11. V. Mezaris, I. Kompatsiaris, and M. G. Strintzis, "Region-based Image Retrieval using an Object Ontology and Relevance Feedback", EURASIP Journal on Applied Signal Processing, Special Issue on Object-Based and Semantic Image and Video Analysis, 2004(6):886-901, June 2004.

12. I. Kompatsiaris, V. Mezaris, and M. G. Strintzis, "Multimedia content indexing and retrieval using an object ontology", in: G. Stamou (ed.), Multimedia Content and Semantic Web Methods, Standards and Tools, Wiley 2004.

13. M. Addis, M. Boniface, S. Goodall, P. Grimwood, S. Kim, P. Lewis, K. Martinez, and A. Stevenson, "SCULPTEUR: Towards a New Paradigm for Multimedia Museum Information Handling”, Proc. of ISWC'2003, LNCS 2870, Springer Verlag 2003.

14. P. Schojer, L. Böszörmenyi, H. Hellwagner, B. Penz, and S. Podlipnig, "Architecture of a Quality Based Intelligent Proxy (QBIX) for MPEG-4 Videos", Proc. of the WWW Conference 2003, pp. 394-402, May 2003.

15. D. Mukherjee, E. Delfosse, J.G. Kim, and Y. Wang, "Terminal and Network Quality of Service", IEEE Transactions on Multimedia, Special Issue on MPEG-21, 2004 (to appear). 